\title{
La guerre dans les médias, les médias dans la guerre en Côte d'Ivoire
}

\author{
Raoul Germain Blé*
}

\section{Résumé}

Cet article propose une réflexion sur un genre particulier de discours médiatique d'information : le journalisme d'opinion très en vue en Côte d'Ivoire depuis la guerre de septembre 2002 qui a coupé le pays en deux parties. Il s’inscrit donc dans une approche constructiviste car il est évident que l’information journalistique procède d’une entité fabriquée. La question des «médias dans la guerre, la guerre dans les médias » constitue un enjeu majeur pour le débat public en le consacrant comme un espace public nouveau dans la formation et l'expression des opinions. Dans le contexte ivoirien, l'information est le paradigme dominant et l'informateur est le journaliste-militant dont la relation à son public se construit sur un mode essentiellement d'appartenance, au sens idéologique du terme. Le discours journalistique ajoute à sa fonction traditionnelle d'informer de nouvelles fonctions de conditionnement et de mobilisation qui en font pleinement un espace idéologique d'adhésion totale.

\begin{abstract}
This paper proposes a reflection on a particular type of informative media discourse: opinion journalism, which has been very prominent in Côte d'Ivoire since the war on September 2002 which split the country into two parts. The paper, therefore, uses a constructivist approach based on the obvious fact that journalisitic information is the product of a created entity. The issue of "the media in the war, the war in the media” is a major issue for public debate, for it is established as a new public space in the formation and expression of opinions. In the Ivorian context, information is the dominant paradigm and the informant is the activist-journalist whose relationship to his/her audience is built on a mode mostly based on membership, in the ideological meaning of the term. The journalistic discourse adds to its traditional function, which is to inform, new conditioning and mobilization functions that make it fully an ideological space of full membership.
\end{abstract}

* Enseignant-chercheur, CERCOM / UFRICA, Université de Cocody-Abidjan.

E-mail : bleraoulgermain@yahoo.fr. 


\section{Introduction}

La Côte d’Ivoire fut longtemps le pays de rêves audacieux, des défis lancés par Félix Houphouët-Boigny, premier Président de la République (1960-1993), dont la préoccupation essentielle fut de donner aux Ivoiriens des conditions meilleures de vie. Son pari le plus osé fut d'affirmer à la face de la nation et du monde entier que la non-violence est le principal facteur de paix et de progrès. Dans un contexte mondial où plus de 500 milliards de dollars USA sont dépensés chaque année pour l'acquisition des armes de guerre, il n'a jamais accepté d'investir dans ce domaine. Ange Barry-Battesti (1995:5) dans l'Atlas de la Côte d'Ivoire, Éditions J.A., fait bien de préciser : « cette politique de paix savamment mise en œuvre par le Président Félix Houphouët-Boigny a été consolidée par un contexte monopartiste rigide ».

La vie étant une dynamique, les partis uniques furent contestés dans tous les pays africains à partir de 1990. Comme une forte envie longtemps contenue, la parole fut soudainement et radicalement libérée sur le continent, avec des fortunes diverses, dans chaque pays. Certains, comme pour mépriser les Africains, y ont vu le mouvement irréversible du vent de l'Europe de l'Est, comme s'ils n'étaient pas capables de faire changer les choses qui environnent et influencent leur quotidien ; ainsi, pour d'autres, il s'agit de l'aboutissement des luttes politiques contre la colonisation qui ont consacré les indépendances. Pour les Africains, la décolonisation fut la première étape d'une longue lutte qu'il fallait consolider par la démocratie en rompant avec la dictature des partis uniques.

Il faut souligner que dans tous les cas, deux phénomènes importants ont marqué la naissance du multipartisme sur le continent noir : Il s'agit des nombreuses conférences nationales et du printemps de la presse, répondant à un besoin d'exister de chaque citoyen par la prise en compte de ses préoccupations, à travers la parole libérée et le droit de vote. Le comportement nouveau des Africains, de ce contexte de prise de conscience, fut une bonne réactivité citoyenne mais mal régulée, faute d'un véritable cadre normatif. Du coup, en ce qui concerne la presse, personne ne put la contrôler, encore moins la maîtriser. Tout s'est passé à une vitesse effrénée comme si elle s'était assignée pour rôle de suppléer le manque de véritable forum.

Pour revenir à la Côte d’Ivoire, le Président Félix Houphouët-Boigny mourut en décembre 1993. Sa succession s'est faite dans une atmosphère difficile, pour deux raisons principales : d'abord, d'un point de vue culturel et socio-identitaire, il appliqua dans sa gestion de la cité, une tradition akan (dont il est originaire) qui veut qu'un chef de son vivant, n'ait pas de successeur connu. Dans ce sens, il préféra «remanier » l'article 11 de la constitution, lequel faisait du Président de l'Assemblée Nationale (lui aussi 
akan) son successeur constitutionnel, en cas de vacance du pouvoir. Ensuite, comme nous l'avons déjà dit, la Côte d'Ivoire, à l'instar de tous les pays africains, avait également renoué avec le multipartisme dans un contexte où l'on compte un peu plus de 60 pour cent d'analphabètes et plus 90 pour cent de la population subissant la pauvreté et la misère sociale.

Il faut cependant ajouter que la longévité politique exceptionnelle de celui qu'on appelait affectueusement « le Père de la nation » avait fini par créer une forte tension relativement à sa succession. Dans ce sens, toutes les tensions sociales longtemps contenues par le monolithisme politique trouvèrent, dans le nouveau système multipartiste, un terreau fertile. Six ans après sa mort, son dauphin constitutionnel, Henri Konan Bédié, en 1999, fut renversé par la junte militaire dirigée par le Général Guéï Robert qui mit en place un gouvernement de transition. Le Général-Président organisa, en octobre 2000, les élections présidentielles (qu'il perdit) et que remporta Laurent Gbagbo.

Le 19 septembre 2002, alors que le Président Laurent Gbagbo effectuait une visite officielle en Italie, il y eut une tentative de coup d'État. De violents affrontements eurent lieu entre l'armée régulière et les rebelles dans les villes d’Abidjan, Bouaké et Korhogo. Le Général Robert Guéï, ancien chef d’État et Émile Boga Doudou, Ministre de l'intérieur dans le gouvernement de Gbagbo furent tués lors de ce putsch manqué. Depuis cette date, la Côte d'Ivoire et sa population sont dans une descente en enfer. Ainsi, ce pays qui fut longtemps la « vitrine » de l'Afrique francophone au sud du Sahara, traverse, aujourd'hui une période douloureuse de son histoire.

Au total, ce qui nous intéresse, en tant qu'enseignant des Sciences de l'Information et de la Communication, c'est ici le degré et la forme d'implication de la presse nationale dans cette guerre. Nous avertissons cependant le lecteur que cet article est le troisième d'une série que nous avons entreprise dans le même ordre d'idée. D’abord, nous avons, dans l'urgence, commencé par « Journalisme civique et réconciliation nationale en Côte d'Ivoire », qui est en réalité la conclusion de la série puisqu'il propose des pistes de réflexion aux fins d'une presse citoyenne, support des politiques de développement, d'émancipation des populations et de cohésion sociale. Ce travail est publié dans le numéro 14/2005 de la revue En-Quête de l'Université de Cocody, à Abidjan (Côte d'Ivoire). Ensuite, le deuxième article, « Médias d'opinion et crise ivoirienne », constitue la partie introductive qui doit aider à la compréhension du concept de crise. Cette réflexion est mise en ligne en mars 2007 dans les enjeux de l'information et de la communication qui est la revue scientifique (électronique) du Groupe de Recherche sur les Enjeux de la Communication (GRESEC) de l'Université de Grenoble, ${ }^{3}$ en France.

Enfin, ce dernier article se situe logiquement en deuxième partie de cet ensemble, comme une étude de cas. 
Ce rappel était nécessaire pour faire comprendre au lecteur les raisons pour lesquelles certaines omissions volontaires ont été opérées dans cet article. L'exercice était difficile quand on sait que les trois textes sont intimement liés, répondant pratiquement à une même méthodologie d'approche, à un même contexte sociopolitique, à une même période et à une même dimension théorique. Ce qui est donc tu, dans l'un des titres, peut être lu dans les autres car les trois articles constituent les différentes parties d'un même système où l'on peut entrer par diverses portes.

\section{Cadre théorique et approche méthodologique}

Une étude sur le journalisme en Côte d'Ivoire couvre un large domaine à la fois pluri et inter disciplinaire qui peut être envisagé dans des perspectives différentes allant d'une vision journalistique au sens strict du terme à un point de vue beaucoup plus global où la pratique du journalisme constitue un des éléments essentiels de lecture et de compréhension des pays africains, en pleine démocratisation.

Depuis 2004, de nombreux ouvrages et publications universitaires, en Côte d'Ivoire, consacrés à l'évolution de la situation socio-politique du pays s'accordent sur l'idée selon laquelle les médias et les journalistes ont une part considérable dans le déchirement du tissu social. Dans ce sens, les entreprises de presse sont confrontées à un environnement incertain à l'origine de bouleversement tant d'un point de vue des valeurs que des pratiques chez les journalistes. Ainsi donc, on constate d'importants changements qui redéfinissent le territoire des médias depuis 1990. Ce phénomène qui s’est accentué avec la pluralité des titres a révélé des considérations de toutes sortes dont les principales sont d'ordre politique, économique, social et culturel.

Dans ce contexte nouveau, on constate des pratiques professionnelles nouvelles ayant donné naissance à deux courants intéressants du point de vue de la recherche : il s'agit du « journalisme d'opinion » et du « journalisme d'information ». Le premier est, en Côte d'Ivoire, le fait des partis politiques qui ont créé leurs journaux afin de maintenir le lien sacré avec leurs militants ; le second est l'œuvre des opérateurs économiques qui ont investi dans ce secteur. Ces derniers, refusant toute compromission, demandent à leurs journalistes-employés de ne pas tremper dans la politique politicienne. Cette recommandation les invite en permanence à l'autocensure.

Dans le premier cas, l'information est le paradigme dominant et l'informateur est le journaliste-militant dont la relation à son public se construit sur un mode essentiellement d'appartenance, au sens idéologique du terme. Le discours journalistique qui est alors un construit social ajoute à sa fonction traditionnelle (d'informer), de nouvelles fonctions de conditionnement, de 
mobilisation, d'identité, d'intégration qui en font pleinement un espace idéologique d'adhésion totale. Un militant lit le journal pour s'informer de l'avis du parti. L'information reçue l'invite à adopter un comportement conforme à la ligne éditoriale, laquelle puise son idéologie dans les directives du parti dont le lecteur-militant et le journaliste-militant sont, tous les deux, membres. Selon Lionel Bellenger (2000:108), « on exprime, par l'effet « band wagon », le fait que l'individu tend à être attiré par le " climat d'opinion » des groupes auxquels il appartient ». Pour la grande majorité des militants, il ne leur est pas donné de critiquer et/ou de remettre en cause (sauf, en interne, de rares fois) les directives de leur parti. On pourra ainsi parler du raz-demarée de l'information car le journaliste d'opinion, de par sa vocation même, manie l'information dont il vit. Cette information savamment construite est partagée par tous les lecteurs sociétaires du parti qui en constituent les réseaux de partenariat, lesquels fonctionnent comme des ethnies ayant leur propre langage, leurs valeurs, leurs intérêts, etc. C’est une évidence que l'article de presse procède d'une construction car son contenu est une entité fabriquée, différente du fait brut. Dans ce sens, on peut avancer que le constructivisme, au sens de la démarche de John Searle, est un point de vue indiqué sur la pratique journalistique. Si au niveau interne, ce type de journalisme repose sur la cohésion du groupe, la solidarité et le sentiment d'appartenance, par contre, en externe, (par rapport aux autres partis), il s'inscrit dans une dynamique d'antagonisme politique où il faut chercher à neutraliser les adversaires. Les conséquences d'une telle évolution se traduisent par les tensions qu'il suscite, par l'absence d'éthique et le non respect des citoyens qui pensent autrement. La coloration ethnique des familles politiques et le taux élevé d'analphabètes favorisent de telles maladresses comportementales. De ce fait, le journalisme d'opinion avec son idéologie d'exclusion, s'inscrit contre l'esprit de la démocratie.

Dans le second cas, il y a deux logiques contradictoires : celle d'un journalisme qui ambitionne de se fixer sur le fait brut mais qui, en même temps, a des contraintes en tant qu'entreprise marchande. Au commencement, ces journaux sont nés pour occuper des espaces abandonnés par les médias d’État, longtemps dépositaires de la pensée unique (de 1960-2000). Malheureusement, comment concilier une logique professionnelle et une logique marchande ? Le professeur américain de sociologie de l'Université de Berkeley (cité par Jean Solbès 1988:31) donne le ton : « les médias sont défensifs parce que dans notre culture politique ils doivent arborer le message de l'objectivité. Ce masque cache l'insécurité des journalistes, tout comme l'insécurité de la profession. Si les liens politiques étaient trop clairement exposés, alors le journaliste serait accusé de prendre parti, ce qui constitue la 
pire chose dans ce milieu... ». En Afrique, les patrons de presse ont la crainte que les gouvernants leur retirent les autorisations de créer des journaux ; concomitamment, les journalistes ont peur de voir retirer leurs cartes de presse par les instances étatiques de régulation des médias. Or, la logique professionnelle commande qu'on dénonce les abus et l'arbitraire des gouvernants et des leaders politiques, pour garantir la crédibilité des journalistes. Selon Rémy Rieffel (2005:40), « toute entreprise médiatique se doit d'abord de convaincre son public que les informations publiées sont sérieuses et fiables ; elle multipliera donc les signes d'authentification... ». Dans ce sens, on peut faire l'hypothèse que le pouvoir des médias en occident, en tant que machine à imposer une ligne de conduite ou de pensée, est relativement faible.

Pour étayer notre propos, nous rappelons qu'aux États-Unis, il existe de nombreuses études sur l'impact et l'influence des médias sur leur public. On peut présenter très schématiquement le bilan de ces travaux en disant qu'ils ont amené certains chercheurs à refuser le schéma trop rigide d'une influence directe et immédiate des médias. Dans l'entre-deux guerres déjà, Harold Lasswell avait résumé le processus de la communication en ces termes : «Qui dit quoi ? À qui ? Dans quelles circonstances ? Avec quels effets ? »

Quelques années plus tard, les travaux de Paul Lazarsfeld ont montré que l'influence des médias n'était pas directe et immédiate, mais plus tôt indirecte et médiate. Elle s'exerce sous la forme d’un flux à deux étages (« two step flow of communication »). Les messages envoyés par les médias sont discutés et évalués à l'intérieur des groupes dont les leaders d'opinion, souvent plus exposés aux médias, exercent une influence déterminante, jouant parfois un rôle de "vigile » (gatekeeper) et peuvent ainsi accepter ou, au contraire, refuser l'entrée des messages dans le groupe. On ne fait donc pas avaler n'importe quoi à n'importe qui, du moins pas, dans une relation de cause à effet : les facteurs contextuels structurent la communication.

Dans le contexte ivoirien, on peut donc, compte tenu du tribalisme et de l'ignorance (la population compte 65 pour cent d'analphabètes), faire l'hypothèse que les médias d'opinion peuvent exercer une influence davantage dans le sens du renforcement des opinions. À ce fait souvent constaté, on peut donner de nombreuses explications : chaque ivoirien choisit de s'exposer au journal dont le contenu correspond davantage à ses idées et à ses opinions. On peut continuer l'hypothèse, en ajoutant que les individus ont tendance à recevoir les informations en fonction de leurs idées et, par conséquent, à choisir, parmi les contenus véhiculés, ceux qui correspondent le plus à ce qu'ils pensent. Enfin, ils garderont davantage en mémoire ce qui semble conforme à leurs opinions et ce qui leur fournit des arguments en faveur de 
leurs idées. Mais cette perception de la presse en occident capitaliste est loin de la réalité africaine où les $3 / 4$ des populations, analphabètes et pauvres, subissent encore, au quotidien, les difficultés de subsistance, si bien que le journalisme « alimentaire » existe et qui se manifeste par la complaisance et la corruption. Bon nombre d'articles sont souvent des publi-reportages qui, comme « un griot », encensent les personnalités du pays. À la suite de Harold Lasswell et Paul Lazarsfeld, nous faisons remarquer que des chercheurs comme Hans Magnus Enzensberger $(1965,1970)$ ou, dans une moindre mesure, T.W. Adorno (1960) estiment que les médias sont en soi des instruments bénéfiques, mais qu'aux mains du système capitaliste, ils autorisent une manipulation des consciences et l'imposition de modèles logiques appartenant à l'idéologie dominante. Mais, de nos jours, cette option ne fait plus souvent l'unanimité. Selon le Professeur Sery Bailly (2003:119) " les médias sont une référence pour juger d'un niveau de patriotisme, d’indifférence ou de bellicisme qui prévaut dans une société ».

La question des «médias dans la guerre », et « la guerre dans les médias » constitue un enjeu majeur pour le débat public en le consacrant comme espace public nouveau dans la formation et l'expression des opinions. Les discours sur l'apprentissage de la démocratie, singulièrement, en ce qui concerne l'intérêt nouveau pour la " chose publique », le multipartisme, la pluralité des organes de presse et l'existence d'une société civile font désormais partie des préoccupations réelles des Africains. Cette prise de conscience condamne la pensée unique longtemps orchestrée par les premiers dirigeants pour s'ouvrir, en ce qui concerne la presse, à la fois sur la pluralité (dimension quantitative) et le pluralisme (dimension qualitative). Dans cet ordre d'idées, on peut dire à la suite de Patrick Charaudeau (2001:8-13) que le discours journalistique se constitue autour de trois axes (corporate, professionnel et commercial). Le premier est en rapport avec le monde politique, le second s'appuie sur une analyse et une interprétation honnêtes des faits bruts. Il concerne tous les publics. Quant au dernier élément, il s’intéresse exclusivement à la dimension marchande, c'est-à-dire économique. Compte tenu de ces précisions, on peut avancer avec Marcel Burger (2005:127) que " les médias produisent aussi bien du discours professionnel du fait qu'ils fondent sans conteste un domaine professionnel (les médias en général), lequel implique l'engagement d'organisations spécifiques (les médias comme entreprises économiques), ainsi que les membres de la catégorie socioprofessionnelle qui les structurent (les journalistes) ». Nous ajouterons que les médias s'adressent principalement à des destinataires considérés comme des consommateurs, se préoccupant de fidéliser ceux-ci pour susciter également l'intérêt des annonceurs investis dans l'information médiatique. 
Une telle visée " commerciale » se situe nécessairement aux confins de l'espace public et constitue dès lors une contrainte de fonctionnement opposée à la contrainte civique.

Dans le cadre de cette étude, l'accent est mis sur le discours journalistique (corporate) qui concerne principalement l'environnement politique en termes de presse d'opinions s'enracinant dans la culture des partis politiques. Cette culture s'exprime en croyance et système de valeurs acceptés comme postulats et référents des idées et comportements collectifs. Elle se traduit en mythes, histoires racontant l'épopée héroïque de la naissance du parti. Par exemple, les journalistes de Le Nouveau Réveil ne peuvent s'empêcher d'évoquer la mémoire de feu Félix Houphouët-Boigny ; ceux de Notre Voie et de Le Patriote en font autant respectivement pour le FPI et le RDR. Si donc la presse d'opinion représente un facteur d'intégration et d'appartenance, sa fonction essentielle est de servir, à l'intérieur du parti, d'espace de régulation dans les rapports individuels et collectifs afin de permettre aux membres et aux sympathisants d'atteindre les objectifs définis par la direction du parti.

On peut lui affecter une seconde mission qui puise son essence dans l'idéologie publicitaire en termes de stratégies d'antagonisme politique (neutraliser les adversaires), de conquête (implantation du parti dans toutes les régions), d'information (fournir des renseignements indispensables aux militants), d'impulsion (inciter à l'adhésion), etc. Dans cette vision, la presse d'opinion utilise toute la culture du capitalisme qui s'inscrit dans le sens du gain. Il faut mobiliser des gens à devenir membres d'un parti, pour en faire des consommateurs achetant, au quotidien, la presse proche dudit parti politique, s'acquittant de la carte d'adhésion et honorant chaque année la cotisation qui sert à « l'entretien » du parti. Ainsi, les membres financent le parti par diverses cotisations.

La presse d'opinion devient une sorte de «bible » dont le contenu ne doit pas être contesté mais en revanche, les journaux du camp adverse sont considérés systématiquement comme nuls et non crédibles. Cette façon de procéder s'inscrit en paradoxe de la démocratie si l'on s'appuie sur Franck Cosson (2005:44) lorsqu'il écrit : « la démocratie rencontre la question de la reconnaissance de l'autre. N'est-il pas légitime de penser que la reconnaissance des différences permet une meilleure expression des libertés individuelles sans que cela mette en péril l'unité de la société démocratique...L'être-commun républicain laisse à chacun la possibilité démocratique que d'être soi ». Nous ajouterons que tout journal, n'importe lequel devrait fonctionner sur le principe de la prise en compte des différentes opinions qui forment la République à travers sa pluralité. Or, la presse d'opinion, partout dans le monde, est exclusive car ne reconnaissant jamais les projets de société du camp adverse, même 
s'ils plaident, de manière pertinente, en faveur de la société, donc de tous les citoyens. Pourtant, la presse dans les nations sous-développées devrait se manifester comme un espace démocratique afin que les choses soient dites (telles quelles) même si elles s'opposent aux intérêts des dirigeants. Espace public au sens de Juergen Habermas (1962), c'est-à-dire, un lieu de rencontre, de structuration et d'échanges des opinions et d'idées afin de favoriser l'émergence d'idées citoyennes dans l'intérêt de la collectivité. Toujours à propos d'espace public et son évolution, il n'est pas vain de lire la contribution de Bernard Miège, dans l'ouvrage collectif, sous la direction d'Isabelle Pailliart (1995:165-175) : « l'espace s’est à la fois perpétué, élargi, voire ses fonctions étendues et pour finir, il s'est fragmenté sur la base d'espaces pluriels ». C'est véritablement le cas de la Côte d'Ivoire, comme nous l'avons déjà évoqué dans une recherche précédente (Blé Raoul Germain 2004:108-118) : il s'agit d'une étude qui porte sur l'émergence d'espaces nouveaux, de taille humaine, où des individus de toutes les conditions sociales expriment librement leur citoyenneté en libérant le verbe dans une oralité dialoguée.

Les propos d'Alex Mucchielli (1998:7-8) cadrent bien avec la situation ivoirienne lorsqu'il dit : " la théorie des processus de communication essaie de rendre compte d'une manière systémique et constructiviste des phénomènes communicationnels qui concourent à l'apparition du sens accompagnant tout élément de communication et, notamment des contextes pertinents dans lesquels, justement, ces phénomènes prennent leur sens, pour les différents acteurs qui sont concernés par ces expressions ». En conclusion de cette dimension théorique, on peut situer notre réflexion sur deux axes complémentaires pour la compréhension des enjeux du journalisme en Afrique. Par rapport aux études sur le développement et l'émancipation, elle tend vers l'apprentissage (mal négocié) de la démocratie. Par rapport à la sociologie des médias, elle s'appuie sur la production journalistique ivoirienne de cette période de la guerre et leur impact sur la société.

De ce qui précède, quel regard peut-on poser sur les médias ivoiriens en cette période de crise depuis 2002 ? Et en particulier sur les trois principaux journaux d'opinions (Notre Voie, Le Patriote et Le Nouveau Réveil) ? Au demeurant comment ont-ils transposé la guerre dans leurs colonnes ? Et quels sont leur sort et leurs responsabilités ? Quant à notre hypothèse, elle indique que parallèlement aux confrontations des soldats sur les champs de guerre, les journaux d'opinion ont transposé la guerre dans leur Une et/ou dans leurs éditoriaux. Des images terribles, de gros titres, des mots forus constituent l'arsenal de cette guerre médiatique dont les dérives ont contribué à amplifier et à prolonger le conflit, car le traitement de l'information n'a pas obéit aux normes éthiques et déontologiques. 
L’objectif de ce travail porte sur une réflexion dynamique visant à :

- tester la ligne éditoriale des quotidiens cités plus haut ;

- identifier et analyser les articles et les images traitant du conflit ;

- analyser le sort des journaux et des journalistes ;

- évaluer l'impact des informations sur la population.

D’un point de vue méthodologique, notre démarche se nourrit de trois parties dont l'articulation colle à la logique même de nos préoccupations. D'abord, nos étudiants de l'Université de Cocody, de l'UCAO et ceux des grandes Écoles ont, par groupe de six, lu les principaux journaux (Fraternité-Matin, Le Nouveau Réveil, Le Patriote, Nord-Sud, L'Inter, Notre Voie, Le Courrier) pour le dépouillement du corpus. Ce travail a été fait pendant six mois de juillet à décembre 2005. Chaque groupe a fait la synthèse du journal que nous lui avons proposé en tenant compte des exigences suivantes :

- travailler sur la base de 6 numéros par semaine de lundi à samedi ;

- chaque étudiant se consacre exclusivement à un numéro dans la semaine ;

- préciser la ligne éditoriale du journal ;

- identifier les articles traitant de ce conflit ;

- analyser leur contenu ;

- dégager de manière nette le brutalisme linguistique et le brutalisme iconique.

Ensuite, à la suite de la contribution de nos étudiants, nous avons entrepris une relecture de quelques articles marquant de manière claire la réalité de la guerre dans les médias et des médias dans la guerre. Mais pour les études de cas, notre corpus repose principalement sur trois journaux (Notre Voie, proche du parti au pouvoir, Le Patriote, proche du RDR/ opposition et Le Nouveau Réveil, proche du PDCI/opposition) sur la période 2005/2006. Puis, à la Une comme dans les pages intérieures, nous avons spécialement choisi d’observer les procédures de mise en scène du chef de l'État et des principaux leaders politiques en empruntant la démarche de Naguénang Wanmé (2004:18-19) :

- «l'image du président Laurent Gbagbo : sa légitimité, ses moyens de lutte, ses rapports avec la France et les autres pays d'Afrique ;

- l'opposition politique : son visage, ses moyens de lutte, sa part dans le conflit ;

- la configuration du conflit : son nom, ses lieux, ses évolutions ;

- la géopolitique : le conflit par rapport au contexte régional africain, la France dans le conflit, l'internationalisation du conflit ».

Pour mieux appréhender la place et le rôle des médias dans la crise ivoirienne, nous avons procédé également à leur analyse de contenu, en nous référant à 
certains éléments de la démarche de Eliseo Veron (1981) et de Dusan Babic (2003). E. Veron porte l'essentiel de son travail sur les aspects topographiques, taxinomiques et l'identité du journaliste qui se dévoile par sa signature. Quant à Dusan Babic, à partir de la question des Balkans dans les médias français, il dégage plusieurs thèses car selon lui, chaque journal a son propre angle par rapport à un même problème. En lisant les principaux quotidiens d'opinion de notre corpus, on se rend compte que la Côte d'Ivoire, dont parlent $L e$ Patriote et Le Nouveau Réveil est " différente » de celle qu'évoque Notre Voie dans ses colonnes et vice-versa.

Il s'agit d'un examen méthodique et quantitatif de notre corpus, en vue d'en tirer ce qu'il possède de significatif par rapport à notre problématique et aux objectifs de notre étude. Enfin, nous avons rencontré un nombre important de journalistes, de responsables d'instances de régulation et d'autorégulation pour avoir les positions des professionnels.

\section{Approche définitionnelle}

Pour que les lecteurs de cette étude soient au même niveau d'information, il est utile de clarifier certains thèmes ou concepts :

\section{La guerre}

Selon le dictionnaire Petit Larousse illustré (1988:477), « La guerre est une épreuve de force entre États, entre peuples et entre partis ». Il s'agit d'une situation dramatique, vécue aujourd'hui par les Ivoiriens, Éthiopiens et les Tchadiens, et hier par des millions d'hommes et de femmes en Sierra-Léone, au Libéria, au Rwanda, en Angola, Congo-Brazzaville, République Démocratique du Congo (anc. Congo-Kinshasa), etc. Nous ajouterons qu'en Côte d'Ivoire où la guerre dure depuis 2002, il s'agit d'un terme rebattu. Point d'ancrage encore du discours de réconciliation. À écouter les différentes manifestations et à lire la presse ivoirienne, on ne peut en effet qu'être convaincu du caractère réel de la guerre.

\section{Les médias}

Le terme de média a été vulgarisé par le sociologue canadien Marshall Macluhan qui l'utilisait comme étant le prolongement de nos sens. De ce point de vue, il peut s'agir d'un crayon, d'une chemise, d'une guitare, etc. De nos jours, nous devons entendre par ce terme, toutes les techniques de diffusion plus large de l'information par la presse, la radio, la télévision, le cinéma et l'affichage. M. Bedouet et F. Cuisiniez (1995:119) ajoutent que «cette réduction [du terme] met en avant la position importante qu'occupent ces médias dans notre société. Les facilités de reproduction donnent au message, en l'amplifiant, une grande résonance sociale ». 


\section{Le brutalisme}

Selon le Petit Larousse illustré (1988:141), « le brutalisme est une tendance architecturale contemporaine qui privilégie l'emploi de matériaux bruts parmi lesquels le béton...la liberté des plans ». Nous ajouterons que le terme est né de «brut », c'est-à-dire, resté à l'état de nature, donc ce qui n'a pas été façonné. Proche de brutalité/brutal qui signifie de manière grossière et violente, il désigne, dans le cadre de notre réflexion, tout excès ou toute outrance. On parlera ainsi de brutalisme linguistique lorsque des excès de langage seront constatés dans les médias de notre corpus. Dans le même ordre d’idées, on parlera de brutalisme « iconique » lorsqu'il sera donné de constater des images horribles ou exécrables.

\section{La guerre dans les médias en Côte d'Ivoire}

La guerre dans la presse est fréquemment mise en scène par les journalistes à travers le choix des mots, des images, des couleurs et leur emplacement, en termes d'occupation de l'espace mais également par les dirigeants politiques aux déclarations "musclées » pour attirer l'opinion publique vers eux. La classe politique ivoirienne est typique de cette forme médiatique depuis 2002.

Le numéro 1788 du 15 septembre 2005 du quotidien Le Patriote donne un aperçu de ce que nous avançons, car il y est écrit à la Une « Gbagbo veut brûler la Côte d'Ivoire ». Il s'agit là d'un brutalisme linguistique, au sens que nous lui conférons. Le verbe brûler, utilisé par Le Patriote signifie (Petit Larousse Illustré 1988:140) « détruire, anéantir, altérer par le feu ou par des produits chimiques ». Ce verbe « brûler » traduit bien l'état de guerre dans le pays. Le journal devient ainsi le prolongement des champs de bataille. Donc le journaliste, en utilisant à dessein ce verbe, tente de prouver l'inconscience de Gbagbo à détruire le pays. Dans ce sens, nous notons que d'un point de vue typographique, chromatique et en ce qui concerne l'investissement de l'espace, ce titre est imprimé en corps gras, en gros caractère et en rouge pour mieux indiquer le danger qui plane sur la Côte d'Ivoire. Son emplacement à la Une lui donne une importance capitale car cette première page correspond dans l'imaginaire de ceux qui connaissent le langage des médias, à la ligne d'attaque des belligérants, au front. Quant au caractère vertical des lettres, il est choisi pour démontrer la volonté de Laurent Gbagbo à anéantir le peuple ivoirien ; c'est pourquoi la couleur rouge est utilisée qui symbolise (négativement) le danger, la peur, la mort ou tout autre événement tragique. Le quotidien Le Patriote étant intégralement imprimé en vert et en blanc, l'introduction de toute autre couleur et en particulier le rouge, participe de sa stratégie de captation.

Sur le plan du brutalisme iconique, on voit, à la page deux, une photo de Laurent Gbagbo dans laquelle il a le visage froncé, la bouche largement ouverte, 
les dents serrées, le nez aplati et le pouce droit tendu vers le bas. Son regard sévère qui scrute l'horizon dans une configuration faciale agressive cadre très bien avec l'idée de quelqu'un qui veut en découdre avec l'ennemi. Le pouce droit baissé vers le bas, en tant que réflexe, annonce une défaite de l'adversaire. Notre propos va dans le même sens que Desmond Mooris, lorsqu'il évoque dans son ouvrage « le langage des gestes » (1994:273) : « le pouce baissé est le contraire du pouce levé qui indique que tout va bien. Il remonte à une habitude de l'ancienne Rome, où il imitait le coup mortel qu'on portait à un gladiateur vaincu dans l'arène. Si la foule voulait la mort de l'homme, elle baissait le pouce, comme pour plonger l'épée dans son corps. Comme le public était assis autour de l'arène, en hauteur, le corps ne pouvait être donné que vers le bas ». De nos jours, le pouce baissé a conservé son sens négatif. En associant à dessein cette image non valorisante du chef de l'État et la phrase suivante «si je quitte le pouvoir, ce sera une guerre civile de dix ans ", Le Patriote nous donne la preuve que la mise en scène de l'écrit et de l'image, souvent utilisés hors de leur contexte d'origine, peut effectivement créer de vives tensions, par la manipulation et la désinformation du public. Les photos représentent une arme puissante dans la guerre médiatique car elles sont utilisées pour anéantir ou ridiculiser : par exemple, les journalistes ivoiriens ont la manie de renverser les photos. Cette façon de procéder participe de la dégradation de l'image de l'adversaire, au même titre que les caricatures et les sobriquets (Woudi de mama (Gbagbo), brave tchê (Alassane Dramane Ouattara) ou N'zuéba (Henri Konan Bédié) qui permettent de dépeindre des situations ou des leaders politiques par la moquerie. Dans ce sens, note Reffiel Remy (2005:180) : « la dramatisation et la théâtralisation de la vie politique sont une autre méthode qui a fait ses preuves depuis quelque temps. Elles consistent à assimiler la politique à un spectacle, à jouer constamment sur les affects aux dépens des programmes, des propositions et des idéologies [...]. Il s'agit à chaque fois de privilégier les effets d'annonce (faire savoir et faire croire qu'on agit) et les effets d'emballage (habiller les mesures prises pour leur donner davantage de visibilité ... ».

Toujours dans le sens du combat, Le Patriote (proche d'Alassane Dramane Ouattara) donne de la voix en ajustant son tir, cette fois, sur le couple présidentiel : "Gbagbo joue avec son destin pendant que Simone réfléchit à son sort ». Ce titre à la fois « compatissant », moqueur et pessimiste se trouve bien illustré par une photo qui présente le couple sous un mauvais jour. Le Président Gbagbo a l'air anxieux et son regard (est) hagard. Cette image est différente de celle d'Alassane Dramane Ouattara qui, lui, présente un visage radieux et serein comme pour traduire qu'il est l'homme de la situation. Le Patriote évoque également le sort de 30000 chômeurs durant 
les six jours (en novembre 2004) d'accrochage entre la Force (française) Licorne et les jeunes patriotes ivoiriens pour annoncer les difficultés qui attendent Gbagbo quant à la gestion de ces citoyens déversés dans la rue.

Aucune guerre ne se gagne sans alliés, note le Président Laurent Gbagbo lors de sa rencontre avec le peuple Akyé en mars 2003. Cette assertion est d'autant plus justifiée que les grandes guerres militaires ont été remportées par des coalitions. En effet, pour vaincre l'Allemagne et ses alliées, la France, l'Angleterre, l'URSS et les USA ont constitué (1939-1945) une coalition forte et solidaire. En Côte d'Ivoire, cette coalition s'est même transposée dans la presse. Pour mieux mener leur combat, les organes proches de l'opposition (RDR, PDCI, Forces Rebelles, MFA, etc.) défendent non seulement les mêmes points de vue, mais sont également parvenus à publier en commun un numéro hors série le 29 novembre 2004, où l'on peut lire les événements de la Côte d'Ivoire aux « colorations » des journalistes de l'opposition. On peut faire donc l'hypothèse qu'il s'agit là d'un puissant arsenal de guerre pour mettre fin au pouvoir de Laurent Gbagbo, en tentant de l'isoler aussi bien sur le plan national, africain, qu'international.

À ce propos, le contrat de représentation qui lie les hommes politiques aux journaux qui les accompagnent dans la conquête du pouvoir est respecté. Dans cet ordre d'idées, on pourrait citer Alphonse Djédjé Mady, Secrétaire Général du PDCI qui harangue son auditoire, en ces ternes, lors d'un meeting : «Si Gbagbo est isolé, le PDCI, quant à lui, présent sur les fronts, se rassemble et que de la sorte, c'est la guerre de tous contre un seul ». Pendant que Fraternité-Matin (quotidien gouvernemental) et Notre Voie (proche du FPI) dénoncent crânement l'attitude belligérante de la force Licorne en condamnant les horreurs et les atrocités perpétrées par celle-ci sur les Ivoiriens, Le Patriote et Le Nouveau Réveil sont préoccupés par autre chose. Oblé Narcisse (DEA : 2005 ISCOM/UCAO) note dans un travail d'évaluation que « dans ce numéro Hors série $n^{\circ} 3$, le Professeur Alphonse Djédjé Mady pense que cette tuerie a été planifiée par le pouvoir en place. Lors d'une déclaration de son parti, il soutient la France et son armée ». Il s'agit là de l'illustration parfaite de la guerre dans les médias en 2004.

D'ailleurs, suite à l'attaque de la ville de Gohitafla, dans le centre-ouest de la Côte d'Ivoire, en zone de confiance, le porte-parole des Armées, dans une mise au point rendue publique le 10 juin 2004, a fustigé la presse dont les écrits sont qualifiés de « tendancieux et sous-tendus d'intentions inavouées ». Il a marqué son mépris par rapport aux auteurs de ces articles dont l'attitude est comparable, selon lui, à celle des terroristes ou des gens sans foi ni loi qui disposeraient d'une arme de destruction massive. 
Cette coalition se continue en 2005 avec le quotidien Le Nouveau Réveil du 15 septembre 2005 qui (s)'annonce dans une autre Une, proche de celle de Le Patriote, où l'on peut lire « Le FPI dans sa logique de guerre ». À la suite de ce titre, il cite Affi N'guessan, président du FPI : « écarter Gbagbo c'est la guerre ». Cette citation est écrite en rouge pour bien marquer le caractère grave et dangereux d'une telle déclaration. Mais pour mépriser son auteur, Le Nouveau Réveil écrit en sous-titre « KKB répond à Affi : « Gbagbo sera écarté, pian ! ». Il faut rappeler que KKB est le président des jeunes du PDCI. Dans cette guerre dans les médias, à forte base psychologique, il faut, pour mobiliser et rassurer ses troupes, mépriser l'adversaire. C’est pourquoi, le PDCI, en laissant le jeune KKB répondre au Président du parti au pouvoir, tente de discréditer ce dernier. D'ailleurs l'interjection purement ivoirienne « pian » qui pourrait signifier dans ce contexte « de toutes les façons » montre que le parti de Konan Bédié ne prend pas au sérieux la menace de l'adversaire.

Le Nouveau Réveil et Le Patriote confirment leur alliance dans leur livraison du 26 octobre 2005 dans laquelle on peut lire (pour le premier) « Gbagbo ancien président » et (pour le second) « Gbagbo : la fin d'un règne nul! ». Les termes de " ancien » et de « fin » montrent bien que ces deux quotidiens se trouvent en harmonie dans leur logique de tourner la page, de l'histoire de la Côte d'Ivoire, sans Laurent Gbagbo. On peut alors avancer avec Erik Neveu (2004:35) que la notion de champ « invite à penser l'espace du journalisme comme un univers structuré pour des oppositions à la fois objectives et subjectives, à percevoir chaque titre et chaque journaliste dans le réseau des stratégies, des solidarités et des luttes qui le lient à d'autres membres du champ... ». Nous ajouterons qu'en France, dans les années 90, c’est le sociologue Pierre Bourdieu qui, le premier, a forgé et utilisé la notion de champ dans ses travaux sur le journalisme. Il s'agit d'un espace social régit par des antagonismes divers dont la principale limite est le recours à la déontologie et à l'éthique.

En outre, il faut ici signaler la composition chromatique qui présente un intérêt particulier. Dans le titre (à la Une) « Gbagbo : la fin d’un règne nul », Le Patriote écrit « Gbagbo » en couleur noire, « la fin d'un règne » en couleur rouge. Rappelons que la couleur noire, dans sa connotation négative signifie enfer, deuil, péché, mort, regret, etc. Nous ajoutons que l’une des clés pour comprendre les mécanismes de la guerre des médias passe nécessairement par la connaissance et la maîtrise du symbolisme des couleurs. Par exemple, le fait d'écrire en noir « Gbagbo », c'est signifier au lecteur que le chef de l' État est définitivement mort politiquement parce qu'il part en enfer (caractérisé par la couleur noire) pour y répondre de ses actes odieux (caractérisés par la couleur rouge). Naturellement, il ne s'agit pas ici de l'enfer au sens théologique 
d'absence de grâce mais de prison, de Tribunal Pénal International et autres contraintes ou privations susceptibles de ternir à jamais son image. Ce titre est renforcé par une photo qui montre Laurent Gbagbo, les yeux baissés, les bras croisés et vêtu d'une chemise locale dont les motifs gais pourraient être confondus (par ses détracteurs) avec des gouttes de sang, comme pour signifier qu'il reconnaît et regrette les actes qui lui sont reprochés.

Notre Voie, proche du Président de la République Laurent Gbagbo, réplique dans sa livraison du mardi 21 septembre 2005, avec une Une ressemblant à celle de ses adversaires : « La France et le bloc rebelle veulent frapper ». Il s'agit d'un gros titre en couleur rouge qui annonce une attaque violente et meurtrière. Ce titre est accompagné d'une photo où l'on voit le président français Jacques Chirac au centre entouré de Henri Konan Bédié à gauche et d'Alassane Dramane Ouattara à droite, tous les deux respectivement présidents du PDCI et du RDR. L'image d'Alasane Dramane Ouattara montre un personnage avec une bouche tordue dans une configuration faciale qui annoncerait dans l'imaginaire de ses adversaires un être sanguinaire, prêt à détruire des vies humaines et à brûler la Côte d'Ivoire pour accéder au pouvoir, à n'importe quel prix. A la guerre comme à la guerre, cette photo est la réplique de celle de Laurent Gbagbo dans Le Patriote du 15 septembre 2005. Quant à Jacques Chirac, il ne présente aucun signe particulier. Il s'agit là d'une photo standard, quelconque, qui signifierait que l'homme Chirac est banal, et qu'il ne mérite aucune considération. En revanche, Notre Voie a présenté curieusement une très belle photo de Henri Konan Bédié, comme pour montrer le visage « mignon » d'un enfant gâté qui, dans l'imaginaire collectif des patriotes, suit naïvement les destructeurs de son propre pays, donc un individu totalement inconscient des enjeux de cette guerre. Sur cette photo, on ne voit aucun souci dans le visage de Henri Konan Bédié, comme si son pays ne traversait aucune crise. Ce discours iconique a été choisi à dessein par la rédaction du quotidien Notre Voie pour situer les responsabilités dans le camp de ce qu'il appelle le bloc rebelle. Un autre élément important, dans ce titre, est l'implication de l'ancienne puissance tutélaire aux côtés des rebelles. La présence française est considérée par les patriotes comme une trahison par rapport à la coopération militaire qui lie les deux pays, et par les politologues comme étant le début de l'internationalisation de la guerre en Côte d'Ivoire.

Dans le même sens, Naguémang Wanmé écrit :

on voit également entrer progressivement en scène des chefs d’État, les diplomaties africaine et française (Laurent Gbagbo, Blaise Compaoré, Dominique de Villepin), des groupes et des espaces (nord/sud, l'ouest de la Côte d'Ivoire) ; on y aborde des questions économiques, géopolitiques, 
historiques, etc. Mais dans son rôle d'information, on constate par ailleurs, que la presse dans son ensemble participe de la nomination du conflit luimême, autant que sa vision structurante en différents mouvements et identités socio-politiques en présence. Ainsi, quelques titres du 20 septembre 2002, lendemain de l'éclatement du conflit sont significatifs : Le Monde informe d'un soulèvement militaire en Côte d'Ivoire, alors que libération indique dans son gros titre que l'État vacille en Côte d'Ivoire. Dès ce deuxième jour du conflit, le dossier de libération contient déjà une interview d'un spécialiste de la Côte d'Ivoire dont le titre est : « Le pouvoir manque de légitimité », et le reportage est une correspondance d'Abidjan. Le Monde attendra un jour de plus, le 21 septembre 2002, titrer qu’ « Une tentative de putsch souligne la fragilité de la Côte d’Ivoire ». Nous ajouterons que le 23 septembre 2002, Libération publie un article intitulé « Un règlement de compte déguisé ». Ce journal avance qu'il n’y a eu « ni mutinerie ni coup d’État mais quelque chose qui ressemble fort à un règlement de compte interne au régime (Wanmé 2004:12).

Ces premiers titres de la presse française, non des moindres, donnent raison à Notre Voie du 21 septembre 2005 qui annonce l'implication de la France aux côtés des rebelles, car à bien comprendre les termes « légitimité » et « fragilité » utilisés par les journalistes français, on découvre leur fonction d'orientation (de leur public) dans le sens habituel d'une dictature (de Gbagbo). Ce contrat de représentation entre la presse française et ses lecteurs est classique de la caricature du continent africain peint de manière grossière et catastrophique.

Notre voie du 26 octobre 2005 réitère son accusation : « Acculée, la France livre Poncet », tout en rappelant dans un gros titre que « l'ONU met le G7 en garde ». Ici Notre Voie fait un contrepoids à la France en indiquant la mise en garde du G7 dont elle est alliée, comme pour signifier que l’ONU, (dans l'entendement commun, à tort ou à raison, est la « chose » des USA) donc les États-Unis d'Amérique sont aux côtés de la république légale (sous contrôle de Laurent Gbagbo) contre la France et ses alliés, les rebelles. La présence de la guerre ne fait aucun doute dans les médias ivoiriens, avec sa forte dose psychologique et sa mise en scène sur fond de manipulation et de désinformation.

\section{Les médias dans la guerre en Côte d'Ivoire}

Les médias ivoiriens ont déserté le terrain de l'information au profit de commentaires et d'éditoriaux partisans. La conséquence d'une telle déviation est marquante avec des rédactions saccagées, des journaux ayant déposé le bilan et des journalistes assassinés. En 2004, Reporters Sans Frontière (RSF) dénombrait 53 journalistes tués dans l’exercice de leur métier, soit le bilan le 
plus lourd. Avec le double, l'année 2005 a battu ce triste record. Et le contexte général de la liberté de la presse se révèle plutôt morose car plus d'un tiers de la population mondiale vit dans un pays où aucun média indépendant n’existe. Au Népal, en Afrique ou en Chine un journaliste peut passer plusieurs années en prison pour un mot ou pour une photo. Quant au contexte ivoirien, en cette période de guerre, il nous livre les données suivantes.

Depuis septembre 2002, Alfred Dan Moussa, Président (d'alors) de l'Observatoire de la Liberté, de la Presse et de la Déontologie (OPELD) note qu'aucun organe de presse n'est en sécurité dans ce pays ». Par exemple, entre le 20 septembre et le 9 octobre 2002, le quotidien Le Patriote (proche du RDR) n'a pu être présent dans les kiosques, parce qu'un danger réel inquiétait sa rédaction, aux dires de Méité Sindou, le rédacteur en chef, d’alors, qui expliquait à la délégation de Reporters Sans Frontière à Abidjan, la situation de son journal. Le 14 octobre 2002, César Etou, rédacteur en chef du quotidien Notre Voie (proche du FPI) est victime d'une agression physique, en fin de journée, dans une rue d'Abidjan. Le même jour, le quotidien Le Nouveau Réveil (proche du PDCI) publie, sur une page entière, un appel à la communauté internationale et à tous les défenseurs des droits de l'homme, annonçant l'enlèvement programmé de Denis Kah Zion, son directeur de publication, et de certains de ses collaborateurs. Par ailleurs, le 16 octobre 2002, vers 9 h 30, des individus dont certains sont armés forcent le portail du siège du groupe Mayama qui édite les quotidiens proches du RDR, saccageant ainsi gratuitement l'ensemble du matériel des rédactions. Le 20 octobre 2002, le domicile du directeur du quotidien Le National, Tapé Koulou, fut attaqué par des hommes armés, tuant sa sœur et son ami. Le 17 octobre 2002, Gael Mocaer, journaliste free-lance, de nationalité française, est interpellé par la Direction de la Surveillance du Territoire (DST), puis libéré le 23 octobre 2002. Quant à Anne Boher, journaliste de nationalité française, travaillant pour le compte de Reuters, elle a été interpellée par les forces de l'ordre à San-Pedro.

De simples arrestations, les choses sont passées à une dimension cruelle, dépassant tout entendement avec la mort de Kloueu Gonseu, journaliste ivoirien à l'AIP, le 19 mars 2003, dont le corps a été retrouvé dans la région de Toulepleu, à l'ouest de la Côte d'Ivoire. Le 23 octobre 2003, Jean Hélène, correspondant de Radio France Internationale en Côte d'Ivoire, de nationalité française, a été tué par balle alors qu'il s’apprêtait à rencontrer quelques opposants sortis de prison. L’homme d'affaires multicartes, de nationalité française, Guy André Kieffer, est porté mystérieusement disparu en septembre 2004.

Dans la partie sous contrôle des Forces rebelles, les journalistes ivoiriens ne sont pas les bienvenus parce que systématiquement soupçonnés d'espions 
pour le compte de Laurent Gbagbo. Dans cette optique, le 20 septembre 2003, Christophe Koffi, correspondant de l'Agence Ivoirienne de Presse au Burkina Faso, se rendant au nord de la Côte d'Ivoire, dans le cadre de ses activités professionnelles, a été arrêté à Korhogo par les chefs rebelles. En outre, les journaux Notre Voie, Le Courrier d'Abidjan et Le Temps, proches du FPI, y sont interdits.

Il va de soi que ces exactions au Nord comme au Sud ont pour seul but d'empêcher les journalistes de faire leur métier, ce qui constitue une entrave à la liberté de la presse, à la liberté d'opinion et d'expression, mais elles présentent aussi des conséquences politiques et économiques. Par exemple, quatre organes (Le Patriote, Le Libéral, Le Front et plus tard Le Nouveau Réveil) ont fait l'objet d'une interdiction de vente dans certaines villes, au motif qu'ils soutiennent la cause des rebelles armés.

Face aux menaces des militants de tout bord politique (les uns dénonçant la collaboration avec les rebelles, les autres dénonçant la pensée unique du pouvoir en place), la société de distribution Edipresse a décidé le 21 octobre 2003, de surseoir à la livraison de journaux dans quelques localités au regard de la tension observée. A l'effet de débattre de la question, les responsables des instances d'autorégulation et de régulation se sont réunis pour trouver une solution d'apaisement. Malheureusement, les menaces vont s'amplifier au point que le 31 octobre 2003, toute la Côte d'Ivoire, sous contrôle gouvernemental, est privée de journaux. Cette situation a entraîné pour bon nombre de rédactions un manque à gagner car un journal est avant tout « une entreprise qui doit respecter les règles du système économique : les frais de production, de distribution et de vente doivent être compensés par des recettes [...] qui sont représentées par les exemplaires vendus et par les insertions publicitaires ", note Touré Dramane Mohamed (2005) dans un travail d'évaluation en DEA, à l'Institut de Communication de l'Université Catholique d'Abidjan. Cette situation de guerre a donc fait subir à la presse ivoirienne énormément de désagréments, ce qui fait dire, dans un communiqué publié dans le quotidien Nord-Sud n³51 du 14 Juillet 2006, à la page 4, par le Groupement des Editeurs de Presse de Côte d'Ivoire (GEPCI), signé de son Président Kah Zion Dénis que « la Presse quotidienne et hebdomadaire est dans un état de déséquilibre structurel et financier sans précédent. En 2001 et 2005 la diffusion a connu une baisse de sept millions et demi d'exemplaires, soit un recul de près de 25 pour cent sur le marché. La situation continue de s'aggraver : le nombre de lecteurs s'est effondré en moyenne de 40 pour cent au cours du premier semestre 2006...». 


\section{Notre Synthèse}

Trois choses marquent notre esprit : le journaliste, son public et le politique qui constituent les acteurs essentiels de cette crise. Mais avant, nous nous permettrons de relever que le phénomène du journalisme d'opinion en Côte d'Ivoire n'a guère été analysé. De cette négligence, on ne saurait s'étonner, car tend à prévaloir, de nos jours, l'étude des structures économiques et sociales et de leurs modifications. Dans cette perspective, le sociologue, le communicologue ou l'historien sont le plus souvent occupés à comprendre comment les crises présentes s'inscrivent dans des conditions objectives et travaillent à les transformer.

Perçu comme un acteur privilégié de la crise, le journaliste d'opinion, lui ne paraît pourtant qu'un figurant dont le comportement est déterminé par celui des hommes politiques qui l'actionnent. Pourtant, si au début des années 90, des journalistes ont contribué à la naissance du multipartisme et de la pluralité des opinions, on note aujourd'hui, de manière nette, qu'ils ne brillent plus particulièrement par leur sens civique, ni par l'obligation déontologique, ni éthique, mais plutôt par leur degré de militance idéologique. Les rédactions ont à leur tête des hommes et des femmes dont l'expression est celle d'autres hommes (hiérarchie politique), réduisant le journaliste à une courroie de transmission. Le journalisme d'opinion devient alors à la fois une incorporation de valeurs et une aliénation qui, comme toute aliénation, n’exclut pas le sentiment d'appartenance à la «tribu ». C'est ce qui fait dire, de manière pertinente, à Virginie Kouassi, Directrice des études à l'Institut des Sciences et Techniques de la Communication (ISTC) à Abidjan, lors d'un séminaire sur le thème "Que pensent et disent les Ivoiriens de la presse et des journalistes ? » : «La plupart des journalistes sont des militants. Leurs écrits sont trop partisans, trop partiaux, trop partiels. L'enthousiasme de départ qu'on a de voir sur le marché une diversité de titres s'évanouit très vite quand on cherche à aller en profondeur des choses. Quand on cherche à s'informer véritablement, quand on veut se faire sa propre opinion des événements et des situations. On a l'impression que vous, journalistes, ne faites pas confiance à notre capacité de jugement, vous voulez tout nous dire mais à votre façon. »

Dans un tel contexte, le journaliste d'opinion n'échappe pas aux mécanismes de façonnement de l'esprit car il forge des réflexes et se soumet à l'autorité de son double paternel et maternel, le leader politique qui finance le journal. Comme chez les Anglais : « qui paie le violon mène la danse ». ! Il s'auto-dirige dans le monde de la servitude consentie car il le sait : seule une application rigoureuse de la ligne du parti permettra d'atteindre l'objectif fixé. De par sa situation privilégiée dans ce contexte des enjeux politiques nouveaux, il se trouve au centre d'un réseau important de communication, 
transmettant aux membres du parti politique les informations, les mots d'ordre de son organisation et son interprétation des événements. En retour, il fait remonter les réactions, les opinions et les sentiments de la base vers la direction du parti afin de vernir la relation d'un " démocratisme », alors qu'en réalité, on en tiendra compte que passablement. Si les directives de son organisation sont déjà préparées et prêtes à être diffusées sous forme de tracts, d'articles ou même de prise de parole auxquels il n'a pratiquement rien à dire, il n'en va pas de même de l'autre opération, dans laquelle il joue un rôle fondamental : il ne transmet jamais au hasard les faits bruts ni la relation des discussions internes, mais il les reconstruit en tenant compte habilement des désirs et des sentiments de son public par rapport à l'idéologie et aux stratégies spécifiques du parti. Souvent, il se comporte en porte-parole de certaines positions, se faisant l'écho (en interne) de la conciliation pour dissiper des malentendus tendancieux ou (en externe) des menaces pour intimider ou répondre aux partis adverses. C'est ce qui fait dire à Bahi Aghi Auguste (2001:130) que " dans les sociétés démocratiques, les médias sont un acteur à part entière des processus sociaux et politiques. Les médias permettent au public d'apprendre des choses dans divers domaines et sont parfois le seul contact qu'une personne puisse avoir avec la réalité politique ». Mais on pourrait se demander si les informateurs sont sincères car leur pouvoir de conviction, si pouvoir de conviction il y a, reposerait beaucoup plus sur celui de l'idéologique que sur des valeurs professionnelles. Le développement de la presse d'opinion en Afrique noire est donc lié à l'apprentissage démocratique, donc il s'agit là d'un processus normal. En revanche, ce qui l'est moins, c'est son contenu sectaire, souvent « tribaliste ». C'est dans ce sens que nous concluons avec Zio Moussa (document tapuscrit non daté que l'auteur a remis à certains de nos étudiants de Licence (2006) lorsqu'il dit : « la dépendance tant financière qu'idéologique des médias des partis et des hommes politiques a servi à préparer, voire à conditionner, dans une certaine mesure, les esprits au conflit armé ivoirien ». On comprend ainsi que la presse s'est constituée en une instance médiatique dans le sens de Charaudeau, cité par Naguémang Wanmé, dans son travail de DEA (2004:145), c'est-à-dire, une instance de production et non un simple "miroir ", à travers un processus de transformation qui consiste à faire passer les faits à travers les mailles du discours qui circulent à travers les schémas de construction et en rapports significatifs avec des occurrences spécifiques, bref une entreprise proche de celle du dramaturge.

\section{Conclusion}

Au terme de cette étude, nous comprenons que les médias d'opinion en Côte d'Ivoire sont des armes de guerre pour marquer des positions stratégiques. Les hommes politiques les actionnent bien souvent aux fins de discréditer ou 
d'attaquer l'adversaire par une escalade de la violence verbale, scripturale, chromatique et iconique. L'apprentissage démocratique, mal négocié, donne ainsi à déplorer l'usage désordonné et mal compris de la liberté et du pluralisme de la presse. Cette situation confirme notre hypothèse selon laquelle les médias ivoiriens ont contribué, à un haut niveau de responsabilité, à la dégradation du tissu social. D'ailleurs, entre journalistes, on note une absence de confraternité qui est sans aucun doute le reflet de l'animosité entre les partis politiques, en dépit des efforts de l'Union Nationale des Journalistes de Côte d'Ivoire (l'UNJCI), pour susciter un sentiment d'appartenance.

La prolifération de la presse d'opinions a des conséquences sociales, économiques et politiques d'une si grande ampleur qu'elle met à risque l'apprentissage démocratique, car elle tire sa force de la vitalité des partis politiques qui la soutiennent et qui transforment leurs membres en lecteurs conditionnés. Par exemple, l'État major d'un parti dicte et applique son idéologie par l'intermédiaire d'une hiérarchie journalistique fortement soumise et bien structurée. Ceci implique une obéissance quasi aveugle de chaque rédacteur aux ordres et consignes venus du parti politique-employeur.

Face à cette situation, les journaux deviennent des armes dangereuses pour le déséquilibre de la communauté. Il est tout à fait normal que les journaux produisent des éditoriaux différents, mais leurs contenus doivent se manifester dans le respect du civisme, de l'éthique, de la déontologie et des valeurs républicaines. C'est dans ce sens que la crédibilité de la presse est à sauver et son professionnalisme considéré dans sa dimension de stratégie structurelle au service d'un développement durable.

\section{Références}

Accardo, A., 1998, Journalistes précaires, Bordeaux : Le Mascaret.

Adorno, T.W., 1960, Television and the patterns of mass culture, Urbana: University of Illinois Press.

Babic, D., 2003, « Une partie des Balkans dans la presse française : différentes approches sporadiques et sélectives, « Cahier de Journalisme, n²12, pp. 88-111.

Bahi, A.A., 2001, « L’effet “Titrologue” : Étude exploratoire dans les espaces de discussion de rues d'Abidjan ", En-Quête, n 8, Université de Cocody, pp. 129-167.

Barel, Y., 1987, La quête du sens, comment l'esprit vient à la cité, Paris : Seuil.

Barthes, R., 1987, L'aventure sémiologique, Paris : Seuil.

Barry-Battesti, A., 1995, Atlas de la Côte d'Ivoire, Paris : Éditions J.A.

Bedouet, M. et Cuisiniez, F., 1995, Vocabulaire de la communication, Paris : ESF.

Bellenger, L., 2000, Du bon usage des médias, Paris : ESF. 
Blé, R.G., 2000, « De la responsabilité éthique des médias et des journalistes en Côte d'Ivoire avant le coup d’État du 24 décembre 1999 », En-Quête, n7, Revue scientifique, Université de Cocody, pp. 27-45.

Blé, R.G., 2004, « Le mythe de la communication », Cahiers congolais de communication, vol.III, $n^{\circ} 1$, pp. 5-21.

Blé, R.G., 2005, « Radio et animation rurale en Côte d’Ivoire, Communication, vol. 23, n² 2, Université Laval, Éditions Nota Bene, pp. 217-236.

Blé, R.G., 2005, « Journalisme civique et réconciliation nationale en Côte d’Ivoire », En-Quête, n 14, Revue scientifique, Université de Cocody, Abidjan, pp. 120135.

Blé, R.G., 2006, « La Responsabilité socio-éthique des Écoles de journalisme en Afrique », Revue du CAMES, nouvelle série B, vol. 007 du $1^{\text {er }}$ semestre, Ouagadougou, pp. 89-96).

Blé, R.G., 2007, "Médias d’opinions et crise ivoirienne », Les Enjeux de l'information et de la communication, Revue électronique du Groupe de Recherche sur les Enjeux de la Communication (GRESEC), Université de Grenoble 3, article mis en ligne le 12 mars 2007.

Bourdieu, P., 1984, Les mensonges du golfe, Paris : Arlea.

Burger, M., 2005, « Les enjeux des discours de débat dans les médias : le cas des débats-spectacle », Communication, vol. 23, n², Université Laval, pp. 125-147.

Champagne, P., 1996, Traitement des malaises sociaux, Cahiers du journalisme, $\mathrm{n}^{\circ} 2$, pp. 9-14.

Charaudeau P., 1997, Le discours de l'information médiatique la construction du miroir social, Paris : Nathan.

Charaudeau, P., 2001, Le discours politique : Les masques du pouvoir, Paris : Éditions Vuibert.

Cosson, F., 2005, La démocratie, Paris : Ellipses marketing, collection Philo-Notions.

De la Haye, Y. et Miège, B., 1985, Journalisme, mode d'emploi (des manières d'écrire l'actualité), Grenoble : Ellug.

Diabi, Y., 1993, « Multipartisme, marketing et communication politique », Revue Tunisienne de Communication, pp. 131-146.

Delforce, B., 1996, « La responsabilité sociale du journaliste : donner du sens », Cahiers de journalisme, $\mathrm{n}^{\circ}$ 2, pp. 17-33.

Delforce, B. et Noyer, J., 1999, « Pour une approche interdisciplinaire des phénomènes de médiatisation : constructivisme et discursivité sociale », Études de Communication, $\mathrm{n}^{\circ} 22$, pp. 13-37.

Derville, G., 1997, Le pouvoir des médias, Grenoble : PUG.

Djereke, J.C., 2003, Fallait-il prendre les armes en Côte d'Ivoire, Paris : L'Harmattan.

Enzensberger, H.M., 1965, Culture ou mise en condition ?, Paris : Julliard, Les Lettres Nouvelles.

Enzensberger, H.M., 1970, « The Consciousness Industry: Constituents of a Theory of the Media », New Left Review, n64, Nov.-Dec., pp. 13-37. 
Esquenazi, J. P., 2002, L'écriture de l'actualité, Grenoble : PUG.

Guery, L., 2001, Précis de mise en page, Paris : CFPJ.

Habermas, J., 1962, L'espace public, Paris : Payot, Collection critique de la politique.

Hunter, M., 1997, Le journalisme d'investigation, Paris : PUF.

La Brosse, R.-d., 2002, « Quelques pistes de réflexions sur le rôle des médias dans les transactions démocratiques », Cahiers du journalisme, n¹0, pp. 232-246.

Lamizet, B. et Silem, A., 1997, Dictionnaire encyclopédique des Sciences de l'Information et de la Communication, Paris : Ellipses.

Le Bohec, J., 1997, Les rapports presse-politique, Paris : L'Harmattan.

Martin-Lagardette, J.L., 2003, Le guide de l'écriture journalistique, Paris : La Découverte.

Miège, B., 2000, Les industries de contenu face à l'ordre informationnel, Grenoble : PUG

Miège, B., 2004, L’information-Communication, objet de connaissance, Bruxelles : De Boeck.

Mooris, D., 1994, Le langage des gestes, Paris : Marabout.

Moumouni, C., 2003, « L’image de l'Afrique dans les médias occidentaux : une explication par le modèle de l'agenda-setting ", Cahiers du journalisme, $\mathrm{n}^{\circ} 12$, pp. 152-158.

Mucchielli, A., 1998, Approches systémiques et communicationnelles, Paris : Armand Colin.

Naguémang, W., 2004, Crise sociale et démocratie en Côte d’Ivoire et au Togo : portraits et mise en scène dans la presse française en 2002 et 2003, DEA SIC, Université de Lille 3.

Neveu, E., 2001, Sociologie du journalisme, Paris : La Découverte.

Neveu, E., 2004, « L’approche constructiviste des problèmes publics : un aperçu des travaux anglo-saxons », Études de communication, n²2, pp. 41-54.

Oblé, N., 2005, Exposé du projet de mémoire de DEA en Communication, Séminaire de recherche, Abidjan : Institut Supérieur de Communication, Université Catholique d'Abidjan.

Pailliart, I., 1995, L'espace public et l'emprise de la communication, Grenoble : Ellug.

Parent-Altier, D., 2003, Approche du scénario, Paris : Nathan.

Ponthieu, G., 1998, Le métier du journaliste en 30 questions, Paris : Dumas.

Reuter, Y., 2003, L'analyse du récit, Paris : Nathan.

Rieffel, R., 2005, Que sont les médias, Paris : Folio Actuel.

Searl, J.R., 1998, La construction de la réalité sociale, Paris :Gallimard.

Sery, B., 2003, Deux guerres de Transition, Abidjan : Educi.

Smith, S., 2003, Négrologie : Pourquoi l'Afrique meurt ? Paris : Calmann-Levy. Solbes, J., 1988, Argent, idéologie, désinformation, Paris : Edition Messidor

Touré, D.M., 2005, Exposé du projet de mémoire de DEA en Communication, Abidjan : Institut Supérieur de Communication, Université Catholique d'Abidjan. 
Tudesq, A.J., 1998, Journaux et radios en Afrique aux XIXe et XXe siècles, Paris : Dumas.

Verschave, F.X., 1999, La Françafrique, Paris : Stock.

Veron, E., 1981, Construire l'événement, les médias et l'accident de Three Miles Island, Paris : Minuit.

Watine, T., 2002, « Pierre Bourdieu : analyse de la construction d'une légende médiatique », Cahiers du Journalisme, n 10 , pp. 11-56. 